\title{
Concomitant essential thrombocythemia with JAK2 V617F mutation in a patient with chronic myeloid leukemia with major molecular response with imatinib and long-term follow-up
}

\author{
KATIA BORGIA BARBOSA PAGNANO ${ }^{1}$, MÁRCIA TORRESAN DELAMAIN ${ }^{1}$, MARIANA MUNARI MAGNUS ${ }^{1}$, \\ JOSÉ VASSALLO ${ }^{2}$, CARMINO ANTONIO DE SOUZA ${ }^{1}$, DAIANE DE ALMEIDA ${ }^{1}$ and IRENE LORAND-METZE \\ ${ }^{1}$ Hematology and Hemotherapy Center, University of Campinas; \\ ${ }^{2}$ Department of Pathological Anatomy, University of Campinas, Campinas, São Paulo, SP 13083-868, Brazil
}

Received December 31, 2014; Accepted January 22, 2016

DOI: $10.3892 / 01.2016 .4631$

\begin{abstract}
The association of chronic myeloid leukemia (CML) with other myeloproliferative neoplasms (MPNs), in particular with the V617F mutation in the Janus kinase 2 (JAK2) gene, is very uncommon, and there are only a few cases reported in the literature. In the present study, the case of a 73-year-old man with CML and persistent thrombocytosis, is reported. The patient achieved a complete cytogenetic response and major molecular response (MR) with imatinib. The patient presented JAK2 V617F mutation, and bone marrow morphology was consistent with essential thrombocythemia. The patient was treated with imatinib and hydroxyurea to control the platelet count, and maintains complete MR with imatinib upon 10 years of follow-up. Although rare, the association of breakpoint cluster region-Abelson rearrangement and JAK2 V617F mutation should be investigated in patients with MPN, since both genetic anomalies may be present at diagnosis or may emerge during treatment, and require different therapeutic approaches.
\end{abstract}

\section{Introduction}

Myeloproliferative neoplasms (MPNs) correspond to a clonal proliferation of hematopoietic cells, resulting in expansion of $\geq 1$ lineages (1). Molecular markers aid to characterize these disorders, and are useful for diagnosis and follow-up of MPNs, since they enable the detection of minimal residual disease, such as breakpoint cluster region-Abelson (BCR-ABL) rearrangement in chronic myeloid leukemia (CML) (1). The Janus

Correspondence to: Dr Kátia Bórgia Barbosa Pagnano, Hematology and Hemotherapy Center, University of Campinas, Carlos Chagas Street 480, Campinas, São Paulo, SP 13083-868, Brazil

E-mail: kborgia@unicamp.br

Key words: chronic myeloid leukemia, myeloproliferative neoplasms, essential thrombocythemia, JAK2 V617F mutation, BCR-ABL, imatinib, molecular response kinase 2 (JAK2) V617F mutation is present in 95\% of patients with polycythemia vera (PV) and in $\sim 50 \%$ of patients with essential thrombocythemia (ET) and idiopathic myelofibrosis (MF) (2-5). The association of CML with other forms of MPN, in particular with the JAK2 V617F mutation, is very uncommon, and there are limited cases reported in the literature (6-8). Pieri et al (9) performed a screening of 314 patients with CML and identified 8 cases $(2.55 \%)$ with concomitant JAK2 V617F mutation.

Despite the rarity of the concomitant detection of BCR-ABL and JAK2 V617F mutation, it is of great importance to recognize and investigate this association, particularly when the disease has an unusual course and the diagnosis of other MPNs may have practical therapeutic consequences. Therefore, in the present study, the case of a patient diagnosed with CML, in whom an ET with JAK2 V617F mutation was detected during the course of CML follow-up, is reported.

\section{Case report}

In March 2003, a 73-year-old male patient was referred to the Hematology and Hemotherapy Center of the University of Campinas (Campinas, Brazil) for a hematological evaluation, due to leukocytosis detected in a routine exam. The patient had no clinical symptoms, and presented a white blood cell count of $49.4 \times 10^{9}$ cells/ 1 [normal range, $5.2-12.4 \times 10^{9}$ cells $/ 1$; $2 \%$ eosinophils (normal range, $0-7 \%$ ), $4.0 \%$ basophils (normal range, $0-1.5 \%$ ), $1 \%$ blasts (normal range, $0 \%$ ) and $2 \%$ promyelocytes (normal range, $0 \%$ )], a platelets count of $607.0 \times 10^{9}$ platelets $/ 1$ (normal range, $150-450 \times 10^{9} / 1$ ) and hemoglobin levels of $12.2 \mathrm{~g} / \mathrm{dl}$ (normal range, 13.5-17.5 g/dl). Diagnosis of CML was confirmed by conventional cytogenetic analysis, using the Giemsa-trypsin-Wright stain banding technique, which revealed the presence of the translocation $\mathrm{t}(9 ; 22)(\mathrm{q} 34 ; \mathrm{q} 11)$ in 20/20 metaphases examined, and by reverse transcription-polymerase chain reaction (RT-PCR), which detected the BCR-ABL rearrangement (b3a2) (10).

In consequence, the patient was treated with interferon alfa-2a (6 million units/day; subcutaneous; Roche Diagnostics, Basel,Switzerland) between April and May 2003, with no hematological response. Thus, treatment with imatinib $(400 \mathrm{mg} / \mathrm{day}$; 


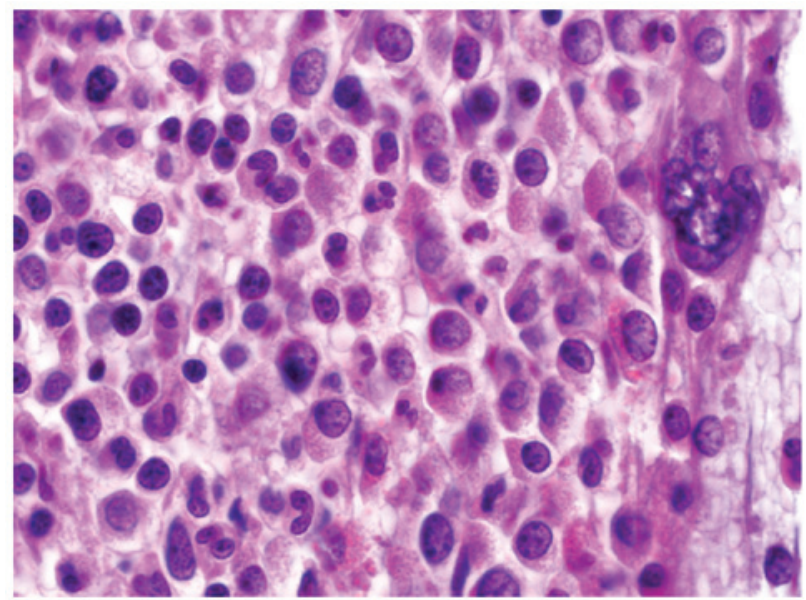

Figure 1. Bone marrow histology at diagnosis revealed chronic myeloid leukemia, according to the hypercellular bone marrow with predominance of the myeloid series and atypical megakaryocytes observed by hematoxylin and eosin staining (Merck Millipore, Darmstadt, Germany) Magnification, x1,000 (Olympus CX31 optical microscope; Olympus America, Inc., Center Valley, PA, USA).

oral; Glivec; Novartis, Basel, Switzerland) was initiated in May 2003. Subsequently, the white blood cell count returned to the normal range, but the platelet count remained elevated. The patient did not achieve a normal platelet count, despite increasing the dose of imatinib to $700 \mathrm{mg} /$ day, but achieved complete cytogenetic remission 6 months later. However, his platelet count increased progressively. Therefore other causes of thrombocytosis were investigated. Ferritin levels were normal and the patient showed increased prostate-specific antigen (PSA) levels (6.7 ng/ml; normal range, 0-4 ng/ml), which suggested prostate cancer. Prostate cancer was diagnosed by prostate biopsy. The patient was treated with local radiotherapy with complete remission. Despite the normalization observed in the PSA levels, the thrombocytosis persisted. In November 2006, the patient achieved a major molecular response (MR) in BCR-ABL levels, but platelets counts remained increased $\left(622 \times 10^{9}\right.$ platelets/1). To further investigate whether other MPNs were involved, the JAK2 V617F mutation was investigated and detected by restriction fragment length polymorphism analysis, as described previously (3), and confirmed by direct Sanger sequencing. The bone marrow biopsies conducted at the time of diagnosis and following treatment for CML were reanalyzed, (Figs. 1 and 2, respectively). The post-treatment biopsy displayed global hypercellularity, increased myeloid:erythroid ratio and hyperplasia of atypical megakaryocytes, with no fibrosis. The morphology of the megakaryocytes was consistent with ET.

The imatinib dose was then decreased to $400 \mathrm{mg} /$ day, as the thrombocytosis was associated with ET and not due to resistant CML. Since then, hydroxyurea $(500 \mathrm{mg} /$ day; oral; Hydrea; Bristol Myers-Squibb, New York City, NY, USA), has been administered concomitantly to control thrombocytosis. In the last follow-up, in December 2015, >10 years following the start of imatinib treatment, the patient maintains a complete cytogenetic response and a deep molecular response [MR 4.5, according to the international scale (11)], in the BCR-ABL transcripts levels detected by RT-PCR,

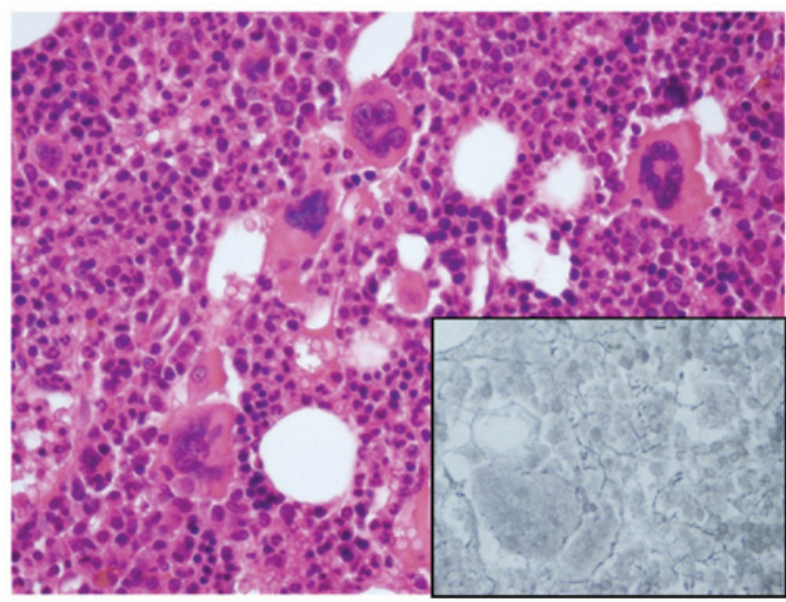

Figure 2. Bone marrow histology following treatment of chronic myeloid leukemia revealed features of essential thrombocythemia, such as proliferation of pleomorphic, hyperlobated megakaryocytes. Erythroblasts and myeloid elements with normal morphology were also present. Hematoxylin and eosin staining (Merck Millipore, Darmstadt, Germany); magnification, x400 (Olympus CX31 optical microscope; Olympus America, Inc., Center Valley, PA, USA). A mild increase in reticulin fibers (Easypath-Erviegas, São Paulo, Brazil) was observed (inset; silver impregnation; magnification, $\mathrm{x} 400$ )

using a previously described technique (12). No thrombotic or hemorrhagic events has occurred.

\section{Discussion}

The association of JAK2 mutations and BCR-ABL rearrangement is infrequent, and was recently reported in association with MF $(6,7,13,14)$ and PV $(8,15,16)$. In a previous report by the present authors, the presence of the JAK2 V617F mutation in CML was reported to be rare, with the present case being the only one detected among 55 cases of CML analyzed (17).

Bee et al (8) reported a case of PV with JAK2 V617F mutation and BCR-ABL rearrangement at diagnosis. In that report, the patient presented a decrease in V617F alleles when the burden of BCR-ABL messenger RNA increased, and reappearance of V617F clones following the administration of imatinib. A similar case was reported by Ursuleac et al (16), where a patient with PV developed CML following 7 years of PV diagnosis. Wahlin et al (18) described a case of ET with emergence of CML during treatment with hydroxyurea. A recent study by Mizutani et al (19) reported the emergence of CML during treatment for ET in a patient following 9 years of diagnosis. By contrast, Véronèse et al (6) reported a case of CML developing ET upon achieving BCR-ABL major MR, similarly to the present findings. In that case, the JAK2 clone was present at low levels at CML diagnosis, while in the present patient, the bone marrow histology was consistent with ET when the mutation was detected, although molecular data regarding the JAK2 mutation was not available at the time of diagnosis.

In conclusion, the present report emphasizes that the presence of persistent or increasing thrombocytosis in CML, which is not responsive to treatment, should always be further investigated. Bone marrow histology may be useful to exclude other malignant diseases or to confirm other MPNs. In the present case, JAK2 V617F mutation analysis was determinant in the 
final characterization of ET associated with CML. Molecular characterization of MPN at the time of diagnosis is necessary to establish a correct diagnosis and to detect concomitant clones. Although rare, the association of ET with CML may be identified at diagnosis or may appear during treatment, and may require to alter the therapeutic approach.

\section{References}

1. Swerdlow HS, Campo E, Harris NL, Jaffe ES, Pileri SA, Stein H, Thiele J and Vardiman JW (eds): WHO Classification of Tumors of Haematopoietics and Lymphoid Tissues. Vol 2. 4th edition. IARC, Lyon, pp23-25, 2008.

2. Baxter EJ, Scott LM, Campbell PJ, East C, Fourouclas N, Swanton S, Vassiliou GS, Bench AJ, Boyd EM, Curtin N, et al; Cancer Genome Project: Acquired mutation of the tyrosine kinase JAK2 in human myeloproliferative disorders. Lancet 365 : 1054-1061, 2005

3. Monte-Mór BDCR, da Cunha AF, Pagnano KBB, Saad ST, Lorand-Metze I and Costa FF: JAK V617F prevalence in Brazilian patients with polycythemia vera, idiopathic myelofibrosis and essential thrombocythemia. Genet Mol Biol 30: 336-338, 2007.

4. Jones AV, Kreil S, Zoi K, Waghorn K, Curtis C, Zhang L, Score J, Seear R, Chase AJ, Grand FH, et al: Widespread occurrence of the JAK2 V617F mutation in chronic myeloproliferative disorders. Blood 106: 2162-2168, 2005.

5. Levine RL, Wadleigh M, Cools J, Ebert BL, Wernig G, Huntly BJ, Boggon TJ, Wlodarska I, Clark JJ, Moore S, et al: Activating mutation in the tyrosine kinase JAK2 in polycythemia vera, essential thrombocythemia, and myeloid metaplasia with myelofibrosis. Cancer Cell 7: 387-397, 2005.

6. Véronèse L, Tchirkov A, Richard-Pebrel C, Ledoux-Pilon A, Fleury J, Chaleteix C, Goumy C, Gouas L, Berger MG, Vago $\mathrm{P}$, et al: A thrombocytosis occurring in Philadelphia positive CML in molecular response to imatinib can reveal an underlying JAK2(V617F) myeloproliferative neoplasm. Leuk Res 34: e94-e96, 2010.

7. Conchon MR, Costa JL, Novaes MM, Dorlhiac-Llacer PE, de Alencar Fischer Chamone D and Bendit I: Simultaneous detection of JAK2 V617F mutation and Bcr-Abl translocation in a patient with chronic myelogenous leukemia. Int J Hematol 88: 243-245, 2008

8. Bee PC, Gan GG, Nadarajan VS, Latiff NA and Menaka N: A man with concomitant polycythaemia vera and chronic myeloid leukemia: The dynamics of the two disorders. Int J Hematol 91: $136-139,2010$
9. Pieri L, Spolverini A, Scappini B, Occhini U, Birtolo S, Bosi A, Albano F, Fava C and Vannucchi AM: Concomitant occurrence of BCR-ABL and JAK2V617F mutation. Blood 118: 344-346, 2011.

10. Cross NC, Melo JV, Feng L and Goldman JM: (1994) An optimized multiplex polymerase chain reaction (PCR) for detection of BCR-ABL fusion mRNAs in haematological disorders. Leukemia 8: 186-189, 1994.

11. Cross NC, White HE, Müller MC, Saglio G and Hochhaus A: Standardized definitions of molecular response in chronic myeloid leukemia. Leukemia 26: 2172-2175, 2012.

12. Ribeiro BF, Vergílio BR, Miranda EC, Almeida MH, Delamain MT, da Silveira RA, de Souza CA, Albuquerque DM, Dos Santos A, Duarte VO, et al: BCR-ABL1 transcript levels at 3 and 6 months are better for identifying chronic myeloid leukemia patients with poor outcome in response to second-line second-generation tyrosine kinase inhibitors after imatinib failure: A report from a single institution. Acta Haematol 134: 248-254, 2015

13. Jallades L, Hayette S, Tigaud I, Johnston A, Coiffier B, Magaud JP and Ffrench M: Emergence of therapy-unrelated CML on a background of BCR-ABL-negative JAK2 V617F-positive chronic idiopathic myelofibrosis. Leuk Res 32: 1608-1610, 2008.

14. Pingali SR, Mathiason MA, Lovrich SD and Go RS: Emergence of chronic myelogenous leukemia from a background of myeloproliferative disorder: JAK2 V617F as a potential risk factor for BCR-ABL translocation. Clin Lymphoma Myeloma 9: E25-E29, 2009.

15. Toogeh G, Ferdowsi S, Naadali F, Alimoghaddam K, Ghavamzadeh A, Shirkoohi R and Ghaffari SH: Concomitant presence of JAK2 V617F mutation and BCR-ABL translocation in a pregnant woman with polycythemia vera. Med Oncol 28: $1555-1558,2011$.

16. Ursuleac I, Colita A, Adam T, Jardan C, Ilea A and Coriu D: The concomitant occurrence of JAK2 V617F mutation and BCR/ABL transcript with phenotypic expression - an overlapping myeloproliferative disorder or two distinct diseases? - case report. J Med Life 6: 34-37, 2013.

17. Pagnano KBB, Almeida D, Delamain MT, De Souza AC and Lorand-Metze I: JAK2 V617F mutation evaluation of chronic myeloid leukemia and acute myeloid leukemia. Rev Bras Hematol Hemoter 31: 169, 2009 (In Portuguese).

18. Wahlin A and Golovleva I: Emergence of Philadelphia positive chronic myeloid leukaemia during treatment with hydroxyurea for Philadelphia negative essential thrombocythaemia. Eur J Haematol 70, 240-241, 2003.

19. Mizutani S, Kuroda J, Shimizu D, Horiike S and Taniwaki M: Emergence of chronic myelogenous leukemia during treatment for essential thrombocythemia. Int J Hematol 91: 516-521, 2010. 